\title{
Nearly subadditive sequences
}

\author{
Zoltán Füredi* Imre Z. Ruzsa ${ }^{\dagger}$
}

October 30, 2018

\begin{abstract}
We show that the de Bruijn-Erdös condition for the error term in their improvement of Fekete's Lemma is not only sufficient but also necessary in the following strong sense. Suppose that given a sequence $0 \leq f(1) \leq f(2) \leq f(3) \leq \ldots$ such that

$$
\sum_{n=1}^{\infty} f(n) / n^{2}=\infty
$$

Then, there exists a sequence $\{b(n)\}_{n=1,2, \ldots}$ satisfying

$$
b(n+m) \leq b(n)+b(m)+f(n+m)
$$

such that the sequence of slopes $\{b(n) / n\}_{n=1,2, \ldots}$ takes every rational number.

When the series (11) is bounded we improve their result as follows. If there exist $N$ and real $\mu>1$ such that (2) holds for all pairs $(n, m)$ with $N \leq n \leq m \leq \mu n$, then $\lim _{n} b(n) / n$ exists.
\end{abstract}

Mathematics Subject Classification: 40A05, 11K65, 05A16.

Keywords: Fekete's lemma, Convergence and divergence of nearly subadditive sequences.

\section{Fekete's lemma on subadditive sequences}

An infinite sequence of reals $a(1), a(2), \ldots, a(n), \ldots$ is called subadditive if

$$
a(n+m) \leq a(n)+a(m)
$$

holds for all integers $n, m \geq 1$.

Every (reasonable) calculus textbook contains Fekete's [8] Lemma as a theorem (or as an exercise, see, e.g., Polya and Szego [12]). It says that if the sequence $\{a(n)\}$ is subadditive, then the sequence $\{a(n) / n\}$ has a limit (possible negative infinity). Moreover, that limit is equal to the infimum,

$$
\lim _{n \rightarrow \infty} \frac{a(n)}{n}=\inf _{k \geq 1} \frac{a(k)}{k}
$$

*Alfréd Rényi Institute of Mathematics, Hungary E-mail: furedi.zoltan@renyi.mta.hu. Research supported in part by the Hungarian National Research, Development and Innovation Office NKFIH, K116769, and by the Simons Foundation Collaboration Grant 317487.

${ }^{\dagger}$ Alfréd Rényi Institute of Mathematics, Hungary E-mail: ruzsa.imre@renyi.mta.hu. Research is supported in part by ERCAdG Grant No.321104 and Hungarian National Foundation for Scientific Research Grant NK104183. 


\section{The standard proof of Fekete's Subadditive Lemma}

Using the subadditivity we get by induction (from $n-k$ to $n$ ) that

$$
a(n) \leq a(k)+a(n-k) \leq 2 a(k)+a(n-2 k) \leq \cdots \leq\lfloor n / k\rfloor a(k)+a(\beta),
$$

where $0 \leq \beta \leq k-1$. (We may define $a(0)=0$ ). This implies that for all $n \geq k \geq 1$

$$
\frac{a(n)}{n} \leq \frac{a(k)}{k}+\frac{\max \{|a(1)|, \ldots,|a(k-1)|\}}{n} .
$$

Therefore

$$
\limsup \frac{a(n)}{n} \leq \frac{a(k)}{k}
$$

This holds for every $k$, so

$$
\lim \sup \leq \inf
$$

implying limsup $=$ inf, so the limit exists.

\section{A remark on large values of $(n, m)$}

Note that the above proof yields that if the subadditivity (3) only holds for $n, m \geq N$, then the limit still exists. We have $a(n) / n \leq a(k) / k$ for all $n \geq k \geq N$ whenever $n / k$ is an integer. In general, we use induction only if both $k$ and $n-k$ is at least $N$, i.e., we choose $\beta \in[k+1,2 k-1]$. Instead of (5) we obtain that for all $n \geq 2 k, k \geq N$

$$
\frac{a(n)}{n} \leq \frac{a(k)}{k}+\frac{\max \{|a(k+1)|, \ldots,|a(2 k-1)|\}}{n} .
$$

This implies (6) and (7) for $k \geq N$. We obtain

$$
\lim _{n \rightarrow \infty} \frac{a(n)}{n}=\inf _{k \geq N} \frac{a(k)}{k} .
$$

\section{Having the threshold $N$ is a true (and nontrivial) extension}

One might be tempted to think that (9) can be easily obtained from the original Fekete's lemma (44). Maybe so, but let us consider the following sequence. Suppose that $2 \leq N \leq n_{1}<n_{2}<n_{3}<\ldots$ are integers such that $n_{i}-N \leq n_{i+1}$. Define for all $i \geq 1$ and positive integer $n$

$$
a(n):= \begin{cases}1 & n \leq n_{1} \\ 1 & n_{i+1}-N \leq n \leq n_{i+1}-2, \\ n / n_{i} & n_{i} \leq n<n_{i+1} \quad\left(\text { but }\left|n-n_{i+1}\right| \notin[2, N]\right) .\end{cases}
$$

This sequence satisfies subadditivity for $m, n \geq N$. Suppose that $\lim \sup n_{i+1} / n_{i}=\infty$. Then the sequence $\{a(n)\}$ does not seem to be easily transformed to a true subadditive one, because there are infinitely many $(x, y)$ pairs with $1 \leq x<N$ and $x+y=n_{i+1}-1$ such that $a(x+y)-a(y)-a(x)=$ $\left(n_{i+1}-1\right) / n_{i}-2$ is arbitrarily large.

(If one prefers an integer sequence, then can observe that $\{\lceil a(n)\rceil\}$ has the same properties). 


\section{Sub-2 sequences by de Bruijn and Erdős}

A sequence $\{a(n)\}$ is called $\mu$-subadditive with a threshold $N((\mu, N)$-subadditive, for short) if

$$
a(n+m) \leq a(n)+a(m)
$$

holds for all integers $n, m$ such that

$$
N \leq n \leq m \leq \mu n
$$

Theorem 1 (de Bruijn and Erdős, Theorem 22. in [4]). Suppose that the sequence $\{a(n)\}$ satisfies (10) for all integers $N \leq n \leq m \leq 2 n$. Then the sequence of slopes $\{a(n) / n\}$ has a limit (possible negative infinity). Moreover, that limit is equal to the infimum,

$$
\lim _{n \rightarrow \infty} \frac{a(n)}{n}=\inf _{k \geq N} \frac{a(k)}{k} .
$$

Actually, they considered the case $N=1$ only. Here we present a greatly simplified proof.

\section{A new proof for Theorem 1}

Fix a $k, k \geq N$. Write $n$ as $n=(\lfloor n / k\rfloor-1) k+\beta$ where $k \leq \beta \leq 2 k-1$. We will show that

$$
a(n) \leq(\lfloor n / k\rfloor-1) a(k)+a(\beta) .
$$

This implies that for all $n \geq 2 k, k \geq N$ inequality (8) holds, implying (9) as in earlier proofs, and we are done.

To prove (12) we need a definition. A sequence of (positive) integers $X:=\left\{x_{1}, x_{2}, \ldots, x_{t}\right\}$ (here $t \geq 1$ ) is called 2 -good if $1 / 2 \leq x_{i} / x_{j} \leq 2$ holds for all $1 \leq i, j \leq t$. If $X$ is a 2 -good sequence of length $t$ and we take two minimal members, $x_{i}, x_{j} \in X$, delete them from $X$ but join $x_{\text {new }}:=x_{i}+x_{j}$, then the new sequence $X^{\prime}:=X \backslash\left\{x_{i}, x_{j}\right\} \cup\left\{x_{n e w}\right\}$ is 2-good as well. Note that the sum of the members of $X$ is the same as in $X^{\prime}$. If the sequence of $\{a(x)\}$ is 2-subadditive then $a\left(x_{n e w}\right) \leq a\left(x_{i}\right)+a\left(x_{j}\right)$ implies that

$$
\sum_{x \in X^{\prime}} a(x) \leq \sum_{x \in X} a(x)
$$

Define the set $X_{\lfloor n / k\rfloor}$ of length $\lfloor n / k\rfloor$ as $\{k, k, k, \ldots, k, \beta\}$. It is obviously a 2-good sequence with sum $n$. Define the sets $X_{t}$ of length $t$ for $\lfloor n / k\rfloor \geq t \geq 1$ by the above rule, $X_{t-1}:=X_{t}^{\prime}$. We obtain $X_{\lfloor n / k\rfloor} \longrightarrow \ldots X_{t} \longrightarrow X_{t-1} \longrightarrow \cdots \longrightarrow X_{1}=\{n\}$. Then (13) gives

$$
a(n)=\sum_{x \in X_{1}} a(x) \leq \cdots \leq \sum_{x \in X_{t}} a(x) \leq \cdots \leq \sum_{x \in X_{\lfloor n / k\rfloor}} a(x)=(\lfloor n / k\rfloor-1) a(k)+a(\beta) .
$$

\section{Sub- $\mu$ sequences with $\mu<2$}

Concerning their result (Theorem 1 above) de Bruijn and Erdős [4] state, maybe somewhat carelessly, that 'It may be remarked that the inequality in (7.1) cannot be replaced by $\mu^{-1} n \leq m \leq \mu n$ 
for any $\mu<2$ '. In their papers [3, 4] they deal with many conditions and sequences, we could not really know what was in their minds, but our first new result is a strengthening of Theorem 1 for all $\mu>1$. We show that their condition can be weakened such that the limit exists if (10) holds only for the pairs $(n, m)$ with $n \leq m \leq \mu n$ for some fixed $\mu>1$.

Theorem 2. Suppose $\mu>1$ and $N \geq 1$ are given. If the sequence $\{a(1), a(2), \ldots\}$ is $(\mu, N)$ subadditive, i.e.,

$$
a(n+m) \leq a(n)+a(m) \quad \forall n \leq m \leq \mu n, n, m \geq N,
$$

then the $\lim _{n \rightarrow \infty} \frac{a(n)}{n}$ exists and is equal to $\inf _{k \geq N} \frac{a(k)}{k}$. (It may be $\left.-\infty\right)$.

For the proof we investigate sequences $\{a(n)\}$ where the subadditivity holds only for a very few pairs $(n, m)$.

\section{Sub-1 ${ }^{+}$sequences}

Given $N \geq 1$ a sequence $\{a(n)\}$ is called $\left(1^{+}, N\right)$ subadditive if the following two inequalities hold for all $n \geq N$.

$$
\begin{aligned}
a(2 n) & \leq a(n)+a(n) \\
a(2 n+1) & \leq a(n)+a(n+1) .
\end{aligned}
$$

Given a sequence $\{a(n)\}$ let $q(n):=\max \left\{\frac{a(n)}{n}, \ldots, \frac{a(2 n-1)}{2 n-1}, \frac{a(2 n)}{2 n}\right\}$.

Lemma 3. Suppose that $N \geq 1$ and the sequence $\{a(n)\}$ is $\left(1^{+}, N\right)$ subadditive. Then for $n \geq N$ the sequence $\{q(n)\}$ is non-increasing, $q(n) \geq q(n+1)$.

We only have to show that $q(n)$ is at least as large as $a(2 n+1) /(2 n+1)$ and $a(2 n+2) /(2 n+2)$. The $1^{+}$subadditivity implies

$$
q(n) \geq\left\{\begin{array}{l}
\frac{a(n+1)}{n+1} \geq \frac{a(2 n+2)}{2 n+2}, \\
\max \left\{\frac{a(n)}{n}, \frac{a(n+1)}{n+1}\right\} \geq \frac{n}{2 n+1} \frac{a(n)}{n}+\frac{n+1}{2 n+1} \frac{a(n+1)}{n+1} \geq \frac{a(2 n+1)}{2 n+1} .
\end{array}\right.
$$

\section{Proof of Theorem 2}

Since the case $\mu \geq 2$ is covered by Theorem 1, we may suppose that $1<\mu<2$. Define the positive integer $k$ by

$$
(1+\mu)^{k-1} \leq 2^{k+1}<(1+\mu)^{k}
$$

Given any $n$ define the sequences $u_{0}, u_{1}, \ldots, u_{k}$ and $v_{0}, v_{1}, \ldots, v_{k}$ as follows.

$$
u_{0}=v_{0}:=n, \quad u_{i+1}:=2 u_{i}, \quad v_{i+1}:=v_{i}+\left\lfloor\mu v_{i}\right\rfloor, \quad(i=0,1, \ldots, k-1) .
$$

We have $u_{k}=2^{k} n$ and $v_{k}>(1+\mu)^{k} n-(1+\mu)^{k} / \mu$. So there exists an $N_{1}$ (depending only from $\mu$ ) such that $2 u_{k} \leq v_{k}$ holds in the above process for every integer $n \geq N_{1}$.

Let $N_{2}:=\max \{N, 1 /(\mu-1)\}$. Then the sequence $\{a(n)\}$ is $\left(1^{+}, N_{2}\right)$ subadditive. Lemma 3 implies 
that $L=\lim _{n \rightarrow \infty} q(n)$ exists. If $L=-\infty$ then $\lim _{n \rightarrow \infty} a(n) / n=-\infty$ as well, and we are done. Since $L<\infty$, from now on, we may suppose that $L$ is a real number.

Choose an (arbitrarily small) $\varepsilon>0$. There exists an $N_{3}$ (depending on $\varepsilon, \mu, N$, and $\left.\{a(n)\}\right)$ such that $q(n)<L+\varepsilon$ for every $n \geq N_{3}$. By the definition of $q$ we get

$$
a(n) / n<L+\varepsilon
$$

for every $n \geq N_{3}$. We are going to show that for $n \geq \max \left\{N_{1}, N_{2}, N_{3}\right\}$

$$
a(n) / n>L+\varepsilon-\varepsilon(1+\mu)^{k} .
$$

Since this holds for every $\varepsilon>0$ the limit $a(n) / n$ exists and is equal to $L$.

To prove (15) we need the following claim which holds for each $i \in\{0,1, \ldots, k-1\}$.

Claim 4. If $a(w) / w \leq L+\varepsilon-\eta$ for every $w \in\left[u_{i}, v_{i}\right]$, then $a(z) / z<L+\varepsilon-\frac{\eta}{1+\mu}$ for every $z \in\left[u_{i+1}, v_{i+1}\right]$.

Indeed, every $z \in\left[u_{i+1}, v_{i+1}\right]$ can be written in the form $z=x+y$ where $x \in\left[u_{i}, v_{i}\right], x \leq y \leq \mu x$. Apply subadditivity for $(x, y)$ and the upper bound $L+\varepsilon-\eta$ for $a(x) / x$ and the upper bound $L+\varepsilon$ for $a(y) / y$. We obtain

$$
\begin{aligned}
\frac{a(z)}{z} & =\frac{a(x+y)}{x+y} \leq \frac{a(x)+a(y)}{x+y} \\
& =\frac{a(x)}{x} \frac{x}{x+y}+\frac{a(y)}{y} \frac{y}{x+y}<(L+\varepsilon-\eta) \frac{x}{x+y}+(L+\varepsilon) \frac{y}{x+y} \\
& =L+\varepsilon-\eta \frac{x}{x+y} \leq L+\varepsilon-\eta \frac{1}{1+\mu} .
\end{aligned}
$$

The end of the proof of Theorem Q Consider any $n$ with $n \geq \max \left\{N_{1}, N_{2}, N_{3}\right\}$. By (14) we have $a(n) / n=L+\varepsilon-h$ for some $h>0$. Consider the intervals $\left[u_{i}, v_{i}\right]$ for $i=0,1, \ldots, k$, where $\left[u_{0}, v_{0}\right]$ consists of a single element, namely $n$. Using Claim 4 we get that $a(x)<L+\varepsilon-h /(1+\mu)^{i}$ for each $x \in\left[u_{i}, v_{i}\right]$ for $1 \leq i \leq k$. Especially, $a(x) / x<L+\varepsilon-h /(1+\mu)^{k}$ for each $x \in\left[u_{k}, v_{k}\right]$. Since $2 u_{k} \leq v_{k}$ we obtain $q\left(u_{k}\right)<L+\varepsilon-h /(1+\mu)^{k}$. But $q\left(u_{k}\right) \geq L$. This implies $h<\varepsilon(1+\mu)^{k}$. We obtained that $a(n) / n=L+\varepsilon-h>L+\varepsilon-\varepsilon(1+\mu)^{k}$ as claimed in (15). This completes the proof of Theorem 2 ,

\section{Nearly subadditive sequences, an error term by de Bruijn and Erdős}

Let $f(n)$ be a non-negative, non-decreasing sequence. deBruijn and Erdős [4] called the sequence $\{a(n)\}$ subadditive with an error term $f$ (or nearly $f$-subadditive, or $f$-subadditive for short) if

$$
a(n+m) \leq a(n)+a(m)+f(n+m)
$$

holds for all positive integers $n, m \geq 1$. The case $f(x)=0$ corresponds to the cases discussed above. 
They showed that if the error term $f$ is small,

$$
\sum_{n=1}^{\infty} f(n) / n^{2} \text { is finite, }
$$

and (16) holds for all $n \leq m \leq 2 n$, then the limit of $\{a(n) / n\}$ still exists.

Let us call a sequence $\{a(n)\}(\mu, N, f)$-subadditive if (16) holds for all $N \leq n \leq m \leq \mu n$. We usually suppose that $f$ is a non-negative monotone increasing real function but we will discuss more general cases as well. Our Theorem 2 yields the following corollary.

Theorem 5. Suppose $\mu>1$ and $N \geq 1$ are given and $f$ is a non-negative monotone increasing real function. If the sequence $\{a(1), a(2), \ldots\}$ is $(\mu, N, f)$-subadditive, i.e.,

$$
a(n+m) \leq a(n)+a(m)+f(n+m) \quad \forall m \leq n \leq \mu m, m, n \geq N,
$$

then the $\lim _{n \rightarrow \infty} \frac{a(n)}{n}$ exists. (It may be $-\infty$ ).

\section{Near subadditivity is really important}

Subadditivity is important, it appears in all parts of mathematics. We all have our favorite examples and applications. But nearly subadditivity is even more applicable, here we mention a few areas.

In the beginning of the Bollobás-Riordan book [2] the de Bruijn-Erdős theorem is listed (as Lemma 2.1 on page 37) among the important useful tools in Percolation Theory. The de Bruijn-Erdös theorem is widely used in investigating sparse random structures, e.g., Bayati, Gamarnik, and Tetali [1] (Proposition 5 on page 4011), Turova [15], or Kulczycki, Kwietniak, and Jian Li [11] concerning entropy of shift spaces.

Also, recurrence relations of type (16) are often encountered in the analysis of divide and conquer algorithms,

$$
a(n+m) \leq a(n)+a(m)+\text { cost of cutting. }
$$

see, e.g., Hsien-Kuei Hwang and Tsung-Hsi Tsai [10]. In Economics it is an essential property of some cost functions that $\operatorname{COST}(\mathrm{X}+\mathrm{Y}) \leq \operatorname{COST}(\mathrm{X})+\operatorname{COST}(\mathrm{Y})$. Similar relations appear in Physics and in Combinatorial optimization (see, e.g., Steele [14]).

Also see, e.g., Capobianco [5] concerning cellular automatas, Ceccherini-Silberstein, Coornaert, and F. Krieger [6] for an analogue on cancellative amenable semigroups.

\section{Proof of Theorem [5 using Theorem 2}

We utilize the proof from [4] (bottom of page 163). For $n \geq N$ define

$$
G(n):=a(n)+3 n\left(\sum_{x \geq n} f(x) / x^{2}\right) .
$$

Then the monotonicity of $f$, the relation $n \leq m \leq 2 n$, and an easy calculation imply that

$$
G(n+m) \leq G(n)+G(m)
$$


whenever (16) holds for $(n, m)$.

Theorem 2 can be applied to $\{G(n)\}$, so we have that the limit

$$
\lim _{n \rightarrow \infty} \frac{G(n)}{n}=\lim _{n \rightarrow \infty}\left(\frac{a(n)}{n}+\left(\sum_{x \geq n} \frac{f(x)}{x^{2}}\right)\right)
$$

exists. Here the last term tends to 0 as $n \rightarrow \infty$ by (17) and we are done.

\section{How large the error term $f(x)$ could be?}

It is very natural to ask how more one can extend the de Bruijn-Erdős theorem concerning $f$-nearly subadditive sequences (the case $\mu=2, N=1$ ). Especially, how large the error term could be?

$f(x)=o(x)$ is necessary

Suppose that $f(n)$ is non-negative and $\lim \sup f(n) / n>L>0$. We can easily construct a sequence $\{a(n)\}$ satisfying (16) for all pairs $m, n \geq 1$ such that $\lim a(n) / n$ does not exist. We do not even use that $f$ is monotone or not.

Given such an $f$ one can find a sequence of integers $1 \leq n_{1}<n_{2}<n_{3}<\ldots$ such that $f\left(n_{i}\right) / n_{i}>$ $L / 2$, and $n_{i+1} \geq n_{i}+2$ for all $i \geq 1$. Define $a(n)=f\left(n_{i}\right)$ if $n=n_{i}$ and 0 otherwise.

$f(x)=o(x)$ is not sufficient

Condition (17) allows $f(x)=O\left(x^{1-c}\right)\left(c>0\right.$ fixed) or even $f(x)=O\left(x /(\log x)^{1+c}\right)$. The first author observed that $f(x)$ could not be $\Omega(x / \log x)$. In 2016 he [9] proposed the following problem for Schweitzer competition for university students (in Hungary). "Prove that there exists a sequence $a(1), a(2), \ldots, a(n), \ldots$ of real numbers such that

$$
a(n+m) \leq a(n)+a(m)+\frac{n+m}{\log (n+m)}
$$

for all integers $m, n \geq 1$, and the set $\{a(n) / n: n \geq 1\}$ is everywhere dense on the real line." (There were two correct solutions: by Nóra Frankl, and Kada Williams and two partial solutions by Balázs Maga, and János Nagy).

\section{deBruijn and Erdös got the best result}

We show that the de Bruijn-Erdős condition (17) for the error term is not only sufficient but also necessary in the following strong sense.

Theorem 6. Let $f(n)$ be a non-negative, non-decreasing sequence and suppose

$$
\sum_{1 \leq n<\infty} f(n) / n^{2}=\infty
$$

Then there exists a nearly $f$-subadditive sequence $b(1), b(2), b(3), \ldots$ of rational numbers, i.e., for all integers $m, n \geq 1$

$$
b(n+m) \leq b(n)+b(m)+f(n+m)
$$


such that the set of slopes takes all rationals exactly once, $\{b(n) / n: n \geq 1\}=\mathbf{Q}$.

The proof is constructive and presented in the next section.

\section{Proof of Theorem 6, a construction}

A typical subadditive function is concave like, e.g., for $a(x)=\sqrt{x}$ we have $\sqrt{x+y} \leq \sqrt{x}+\sqrt{y}$ (for $x, y \geq 0)$. The main idea of the construction for Theorem 6 is that a nearly $f$-subadditive sequence $\{a(n)\}$ could be (strictly) convex with $\lim _{n \rightarrow \infty} a(n) / n=\infty$.

\section{A convex $f$-subadditive function}

Claim 7. Suppose that $f(n)$ is a non-negative, non-decreasing sequence, $0 \leq f(2) \leq f(3) \leq \ldots$ Define $f(1)=a(1)=0$ and in general let

$$
a(n):=n\left(\sum_{i=1}^{n} \frac{f(i)}{i^{2}}\right) .
$$

Then the sequence $\{a(n)\}$ is nearly $f$-subadditive, it satisfies (16).

Proof. Write down the definition of $a(n)$, simplify, use the monotonicity of $f$, finally the estimate $\left(\sum_{u<i \leq v} 1 / i^{2}\right)<(1 / u)-(1 / v)$ (for integers $\left.1 \leq u<v\right)$. We obtain

$$
\begin{aligned}
a(n+m) & -a(n)-a(m) \\
& =n\left(\sum_{i \leq n+m} \frac{f(i)}{i^{2}}\right)+m\left(\sum_{i \leq n+m} \frac{f(i)}{i^{2}}\right)-n\left(\sum_{i \leq n} \frac{f(i)}{i^{2}}\right)-m\left(\sum_{i \leq m} \frac{f(i)}{i^{2}}\right) \\
& =n\left(\sum_{n<i \leq n+m} \frac{f(i)}{i^{2}}\right)+m\left(\sum_{m<i \leq n+m} \frac{f(i)}{i^{2}}\right) \\
& \leq n f(n+m)\left(\frac{1}{n}-\frac{1}{n+m}\right)+m f(n+m)\left(\frac{1}{m}-\frac{1}{n+m}\right)=f(n+m) .
\end{aligned}
$$

Claim 8. The above sequence $\{a(n)\}$ defined by (19) is non-negative and convex, i.e., for $n \geq 2$ we have

$$
a(n) \leq \frac{a(n-1)+a(n+1)}{2} .
$$

Proof. We have

$$
\begin{aligned}
a(n-1)+a(n+1)-2 a(n) & =(n-1)\left(\sum_{i \leq n-1} \frac{f(i)}{i^{2}}\right)+(n+1)\left(\sum_{i \leq n+1} \frac{f(i)}{i^{2}}\right)-2 n\left(\sum_{i \leq n} \frac{f(i)}{i^{2}}\right) \\
& =\frac{f(n+1)}{(n+1)}-(n-1) \frac{f(n)}{n^{2}} \geq \frac{f(n+1)}{(n+1) n^{2}} \geq 0 .
\end{aligned}
$$

\section{The end of the proof of Theorem 6}


In this section $\{f(n)\}$ is given by Theorem [6, and $\{a(n)\}$ is the well-defined nearly $f$-subadditive,

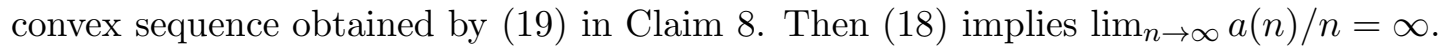

For the rest of the proof the main observation is the following: If $c(1) \leq c(2) \leq c(3) \leq \ldots$ is a monotone sequence, and $\{a(n)\}$ is $f$-subadditive, then

$$
b(n):=a(n)-c(n) n \text { is } f \text { subadditive as well. }
$$

Indeed,

$$
\begin{aligned}
& b(n+m)-b(n)-b(m)-f(n+m) \\
& \quad=[a(n+m)-c(n+m)(n+m)]-[a(n)-c(n) n]-[a(m)-c(m) m]-f(n+m) \\
& =[a(n+m)-a(n)-a(m)-f(n+m)]+(c(n)-c(n+m)) n+(c(m)-c(n+m)) m \leq 0 .
\end{aligned}
$$

Let $r_{1}, r_{2}, r_{3}, \ldots$ be an enumeration of $\mathbf{Q}$. We will define a sequence $1 \leq n_{0} \leq n_{1} \leq n_{2} \leq \ldots$ and simultaneously $\{c(n)\}$ (and thus $\{b(n)\}$ as well) such that

(D) the slopes $\left\{b(n) / n: 1 \leq n \leq n_{i}\right\}$ are all distinct and rational, and

(R) $\quad r_{i} \in\left\{b(n) / n: 1 \leq n \leq n_{i}\right\}, \quad(i \geq 1)$.

We proceed by induction on $i$. Let $n_{0}$ be the smallest $x \geq 1$ such that $f(x)>0$. Equation (18) implies that $1 \leq n_{0}<\infty$. Choose $c(1) \leq \cdots \leq c\left(n_{0}\right)$ arbitrarily such that the fractions $b(x) / x=$ $(a(x)-c(x) x) / x$ are all rationals and they are all distinct. Since these are finitely many constraints of the form

$$
\frac{a(x)}{x}-c(x) \neq \frac{a(y)}{y}-c(y) \quad 1 \leq x \neq y \leq n_{0}
$$

and the set $\mathbf{Q}$ is everywhere dense on $\mathbf{R}$, one can easily choose appropriate $c(x)$ 's.

If $n_{0}, n_{1}, \ldots, n_{i}$ has been already defined (satisfying properties $(\mathrm{D})$ and $(\mathrm{R})$ ) then proceed as follows.

If $r_{i+1} \in\left\{b(x) / x: 1 \leq x \leq n_{i}\right\}$, then let $n_{i+1}:=n_{i}$.

If $r_{i+1} \notin\left\{b(x) / x: 1 \leq x \leq n_{i}\right\}$ then define $n_{i+1}$ as the smallest integer $x$ satisfying

$$
x>n_{i}, \quad \frac{a(x)}{x}-c\left(n_{i}\right)>r_{i+1} .
$$

Such $x$ exists. Let $c\left(n_{i+1}\right):=\frac{a\left(n_{i+1}\right)}{n_{i+1}}-r_{i+1}$. It follows that $c\left(n_{i}\right)<c\left(n_{i+1}\right)$. Then select $c(x)$ for integers $x$ with $n_{i}<x<n_{i+1}$ such that the values of $a(x) / x-c(x)$ are all rationals, distinct from each other, have no common values with $\left\{b(n) / n: 1 \leq n \leq n_{i}\right\} \cup\left\{r_{i+1}\right\}$ and also $c\left(n_{i}\right) \leq c\left(n_{i}+1\right) \leq \cdots \leq c\left(n_{i+1}\right)$. These are finitely many conditions but $c\left(n_{i}\right)<c\left(n_{i+1}\right)$ and $\mathbf{Q}$ is everywhere dense, so the induction step can be done. This completes the construction.

\section{Conclusion, problems}

Let $X \subseteq \mathbf{N} \times \mathbf{N}, f: \mathbf{N} \rightarrow \mathbf{R}$. The sequence $\{a(n)\}$ is $(X, f)$-subadditive if $a(m+n) \leq a(n)+$ $a(m)+f(n+m)$ holds for $(n, m) \in X$. We have found conditions for $X$ and $f$, strengthening the original Fekete's lemma and its de Bruijn-Erdős generalization, which ensure that $\lim a(n) / n$ exists. Certainly further thinning of $X$ are possible. We mention two of these problems. 
Is it possible to replace the constraint $n \leq m \leq \mu n$ in Theorem 2 by some condition like $n \leq m \leq$ $n+r(n)$ where $r(n)=o(n)$ some slow growing function? (Probably not).

What is the structure of $1^{+}$subadditive sequences? Can we tell more than Lemma 3]?

Finally, it is well-known that if $a(x)$ is a measurable subadditive function $a:(0, \infty) \rightarrow \mathbf{R}$, then

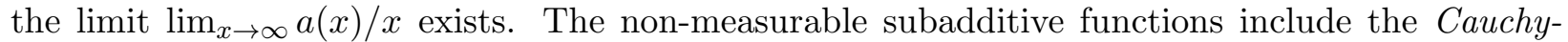
functions which do not have limits, and are far from linear. This is a large field of analysis, and alos in number theory concerning additive functions. There are many results and questions, see, e.g., [7, 13, 16]. 


\section{References}

[1] Mohsen Bayati, David Gamarnik, and Prasad Tetali: Combinatorial approach to the interpolation method and scaling limits in sparse random graphs. Ann. Probab. 41 (2013), 4080-4115.

[2] Béla Bollobás, and Oliver Riordan: Percolation. Cambridge University Press, New York, 2006. $\mathrm{x}+323 \mathrm{pp}$.

[3] N. G. de Bruijn, and P. Erdős: Some linear and some quadratic recursion formulas, I. Indag. Math. 13 (1951), 374-382.

[4] N. G. de Bruijn, and P. Erdős: Some linear and some quadratic recursion formulas, II. Indag. Math. 14 (1952), 152-163.

[5] S. Capobianco: Multidimensional cellular automata and generalization of Fekete's lemma. Discrete Math. Theor. Comput. Sci. 10 (2008), 95-104.

[6] T. Ceccherini-Silberstein, M. Coornaert, and F. Krieger: An analogue of Fekete's lemma for subadditive functions on cancellative amenable semigroups. J. Anal. Math. 124 (2014), 59-81.

[7] P. Erdős: On the distribution function of additive functions. Ann. of Math. 47 (1946), 1-20.

[8] M. Fekete: Über die Verteilung der Wurzeln bei gewissen algebraischen Gleichungen mit ganzzahligen Koeffizienten. Mathematische Zeitschrift 17 (1923), 228-249. Also see, e.g., in [12].

[9] P. Frenkel: The 2016 Miklós Schweitzer Memorial Competition in Mathematics. October $24-$ November 2, 2016. (Organized by the Bolyai Society, Hungary). Problem 4 was proposed by Z. Füredi. See: http://www.bolyai.hu/SCHWEITZER/angol2016.pdf

[10] Hsien-Kuei Hwang, and Tsung-Hsi Tsai: An asymptotic theory for recurrence relations based on minimization and maximization. Theoret. Comput. Sci. 290 (2003), 1475-1501.

[11] M. Kulczycki,D. Kwietniak, and Jian Li: Entropy of subordinate shift spaces. Amer. Math. Monthly 125 (2018), 141-148.

[12] G. Pólya, and G. Szegö: Problems and theorems in analysis, volume 1. Springer-Verlag, New York (1976). (Originally: Aufgaben und Lehrsätze..., Springer, Berlin, 1925, vol. 1, p. 17.)

[13] I. Z. Ruzsa: Additive functions with bounded difference. Period. Math. Hungar. 10 (1979), $67-70$.

[14] J. M. Steele: Probability theory and combinatorial optimization. CBMS-NSF Regional Conference Series in Applied Mathematics 69, SIAM, Philadelphia, PA, 1997. viii+159 pp.

[15] Tatyana S. Turova: The largest component in subcritical inhomogeneous random graphs. Combin. Probab. Comput. 20 (2011), 131-154.

[16] E. Wirsing: A characterization of $\log n$ as an additive arithmetic function. 1970 Symposia Mathematica, Vol. IV (INDAM, Rome, 1968/69) pp. 45-57. Academic Press, London 